\title{
Computerized Method for Automatic Evaluation of Lean Body Mass from PET/CT: Comparison with Predictive Equations
}

\author{
Tao Chan
}

Department of Diagnostic Radiology, University of Hong Kong

CT has become an established method for calculating body composition, but it requires data from the whole body, which are not typically obtained in routine PET/CT examinations. A computerized scheme that evaluates whole-body lean body mass (LBM) based on CT data from limited-whole-body coverage was developed. The LBM so obtained was compared with results from conventional predictive equations. Methods: LBM can be obtained automatically from limited-whole-body CT data by 3 means: quantification of body composition from CT images in the limited-whole-body scan, based on thresholding of CT attenuation; determination of the range of coverage based on a characteristic trend of changing composition across different levels and pattern recognition of specific features at strategic positions; and estimation of the LBM of the whole body on the basis of a predetermined relationship between proportion of fat mass and extent of coverage. This scheme was validated using 18 whole-body PET/CT examinations truncated at different lengths to emulate limited-whole-body data. LBM was also calculated using predictive equations that had been reported for use in SUV normalization. Results: LBM derived from limited-whole-body data using the proposed method correlated strongly with LBM derived from whole-body CT data, with correlation coefficients ranging from 0.991 (shorter coverage) to 0.998 (longer coverage) and SEMs of LBM ranging from 0.14 to $0.33 \mathrm{~kg}$. These were more accurate than results from different predictive equations, which ranged in correlation coefficient from 0.635 to 0.970 and in SEM from 0.64 to $2.40 \mathrm{~kg}$. Conclusion: LBM of the whole body could be automatically estimated from CT data of limited-whole-body coverage typically acquired in PET/CT examinations. This estimation allows more accurate and consistent quantification of metabolic activity of tumors based on LBM-normalized standardized uptake value.

Key Words: lean body mass; standardized uptake value

J Nucl Med 2012; 53:130-137

DOI: 10.2967/jnumed.111.089292

$\mathbf{P}$ for different

Received Feb. 16, 2011; revision accepted Aug. 17, 2011.

For correspondence or reprints contact: Tao Chan, Room 406, Block K, Queen Mary Hospital, Pok Fu Lam, Hong Kong.

E-mail: taochan@hku.hk

Published online Nov. 29, 2011.

COPYRIGHT @ 2012 by the Society of Nuclear Medicine, Inc. used radiopharmaceutical in PET is ${ }^{18} \mathrm{~F}-\mathrm{FDG}$, a glucose analog that accumulates in cells consuming glucose, including most cancer cells. The current clinical practice of PET interpretation is based primarily on qualitative visual assessment. However, quantitative evaluation of metabolic activity is an important adjunct for tumor detection, assessment of disease, and prediction and monitoring of treatment response. Standardized uptake value (SUV), defined as measured radioactivity concentration in tissue divided by injected dose per unit volume of distribution, is the most widely used method for quantitative assessment of clinical PET. The volume of distribution can be total body weight, lean body mass (LBM), or body surface area. SUV normalized by LBM is becoming a popular technique because the value thus obtained is less variable between individuals of different body weights (1-2). Also, SUV normalized by LBM has also been advocated as the preferred method of quantifying metabolic activity for treatment response assessment in clinical trials (3).

Because LBM cannot be conveniently measured, it is derived by predictive equations using some or all of the parameters of height, body weight, sex, and age. But a previous study has shown considerable variations between such formulae for estimation of LBM. It also found that 7 of the 8 evaluated formulae, including 4 that had been used for SUV normalization in the literature, were statistically different from LBM derived from dual-energy x-ray absorptiometry (4). Although additional tests for measurement of LBM may not be practical, the CT data from a PET/CT examination are readily available for accurate evaluation of LBM. Because fat and other tissues vary in $\mathrm{x}$-ray attenuation because of their different densities and chemical compositions, they can be distinguished from one another by their characteristic range of Hounsfield units on CT. In fact, CT has become a standard method for evaluating body composition, with which other techniques, including dual-energy x-ray absorptiometry, are compared (5).

A practical limitation of using the CT method is that LBM can be directly measured over only the range of the body covered in the PET/CT examination. Since most whole-body PET/CT examinations for most clinical indications cover only the range from skull base to upper thighs, it remains to be determined if LBM for the whole person over which ${ }^{18} \mathrm{~F}$-FDG distributes can be accurately estimated from the limited coverage. This paper aims to 
introduce a computerized scheme that was developed to determine LBM obtained from CT of limited-whole-body examinations, and to compare the results so obtained with LBM derived from predictive equations.

\section{MATERIALS AND METHODS}

All PET/CT examinations of adult patients covering head to toe performed over a 3-y period from October 2007 to September 2010 were retrospectively retrieved from the PACS system of the University of Hong Kong. Institutional review board approval was obtained from the university for this Health Insurance Portability and Accountability Act-compliant study. Informed consent was waived.

All examinations were performed on the same machine (Discovery VCT;GE Healthcare) using the same standard protocols. Patients fasted for $6 \mathrm{~h}$ before the examination. A 64-slice multidetector CT scan was obtained (field of view, $50 \mathrm{~cm}$; matrix, $512 \times 512$; slice thickness, $0.625 \mathrm{~mm}$ reconstructed to be $2.5 \mathrm{~mm}$; spiral CT pitch, 0.984:1; gantry rotation speed, $0.5 \mathrm{~s}$ ). An ${ }^{18} \mathrm{~F}-\mathrm{FDG}$ dose of 222-370 $\mathrm{MBq}$ (adjusted according to patient weight) was given intravenously; after an uptake time of $60 \mathrm{~min}$, whole-body PET was performed in around $20 \mathrm{~min}$ (field of view, $70 \mathrm{~cm}$, matrix, $128 \times 128$; slice thickness, $3.27 \mathrm{~mm}$; 6-7 bed positions; 3 min per bed position). Attenuation correction for PET data using CT images was performed, and images were reconstructed using an ordered-subset expectation maximization iterative algorithm (14 subsets and 2 iterations).

There were 2 types of CT protocols, selected at the discretion of the referring physicians in consultation with the duty radiologists. The first was a normal-dose $(120 \mathrm{kV}, 200-400 \mathrm{mAs})$ contrastenhanced CT scan, obtained $90 \mathrm{~s}$ after injection of a nonionic low-osmolar iodinated contrast agent containing $370 \mathrm{mg}$ of iodine per milliliter (Iopamiro 370; Bracco Imaging). The second was a low-dose (120 kV, 80-100mAs) unenhanced CT protocol.

The computerized scheme for automatic determination of LBM from limited-whole-body CT data is illustrated in Figures 1-3 and outlined in the following sections.

\section{Calculation of Fat Mass from CT}

Fat mass from all images of any limited-whole-body scan was calculated. First, the body had to be segmented from the original image, as the images also include unrelated scanned objects such as the blanket, clothing, and scanning couch. Because the blanket and clothing were thin sheets and the scanning couch was a hollow structure, they appeared as thin lines on the transverse images. Such thin lines were readily removed using morphologic opening operations. In addition, the CT dataset was denoised using standard median filtering, which has the advantage of preserving edges between regions. The area of different body constituentsfat, soft tissues, and bone-were automatically segmented from the CT images on the basis of characteristic attenuation, using the ranges proposed in an established technique (6). Then, mass attributable to different types of tissues was calculated by multiplying their areas from all images by slice thickness and the known densities of these tissues (7). For example, normal fat shows a characteristic range of attenuation of -190 to -30 Hounsfield units and an average density of $0.923 \mathrm{~g} \cdot \mathrm{mL}^{-1}$.

The limited-whole-body fat mass was then used to estimate the fat mass from the whole body, extending to parts that were not scanned. This estimation was made possible by automatic determination of the specific range of coverage in any examination, in combination with the reference that related the range of coverage to its proportional contribution of fat mass to the whole body.

\section{Automatic Determination of Coverage}

The levels of top of thorax and bottom of pelvis were automatically determined on the basis of the characteristic trend of changing body composition derived from CT data, refined by locating specific patterns of changing air and bone distributions at these 2 levels.

First, the thorax was identified as the portion of the trunk that contained a large volume of air; this defined the cranial direction in a limited-whole-body scan and the opposite as the caudal direction. Moving craniad from the lung, the apex of the lunghence, top level of thorax —was identified by finding the sections where the area of air dropped sharply. The exact level was defined as the area where disjoint air density regions on both sides of the midline dropped to zero, with only an air density region remaining near the midline (air in trachea or esophagus). On the other hand, moving caudad from the trunk, the area of bony content would

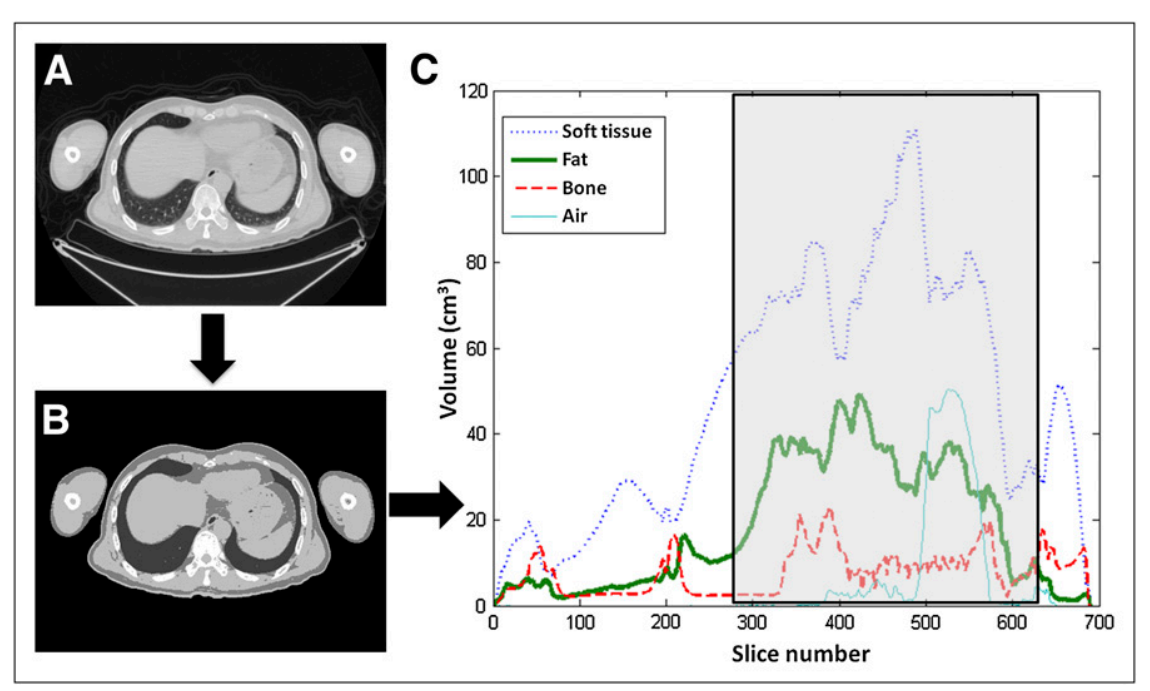

FIGURE 1. Determination of body composition from CT data. (A) First, body is segmented from original image by removing couch and blankets using morphologic operations, by virtue of their thin linear configurations. (B) Then, body is segmented into its constituent tissues, namely bone, soft tissue, fat, and air, based on their characteristic attenuation, represented by 4 shades of gray. (C) Volumes of soft tissue, fat, bone, and air are calculated, with their changing trend along length of body, from foot at image 0 to head at around image 700 in this particular example. Total-body fat mass (hence LBM) can readily be determined by calculating area under fat line when data from whole body are available. The challenge is to accurately estimate whole-body fat mass from data of variable limited coverage, for example, shaded area in $\mathrm{C}$. 


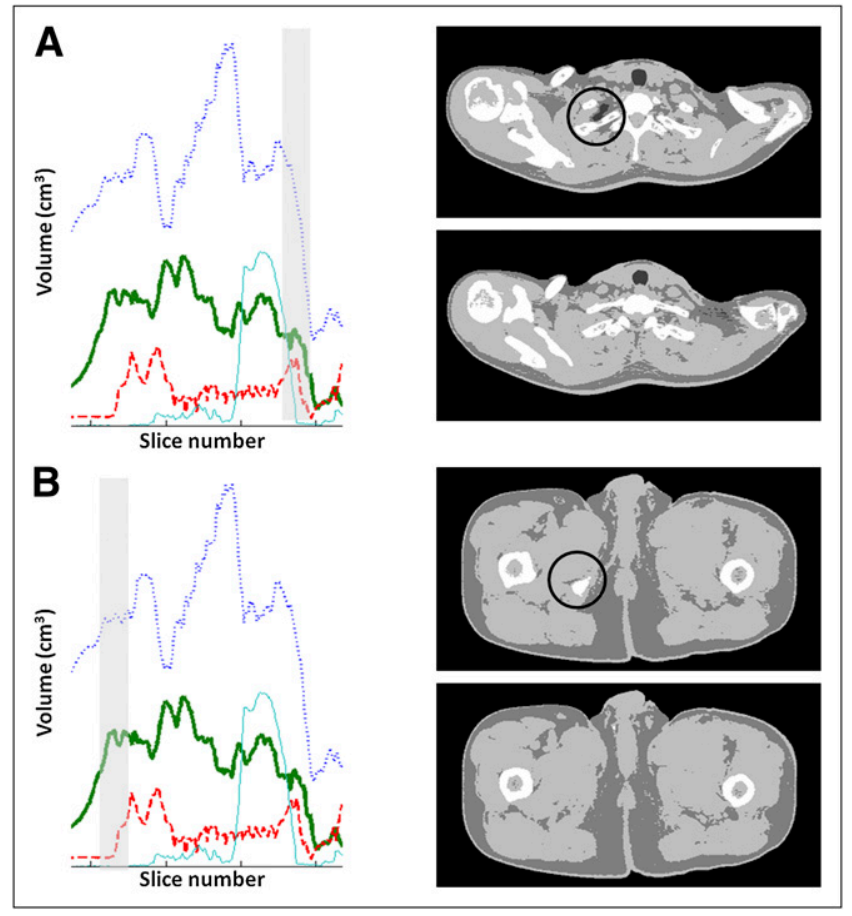

FIGURE 2. Changing trend of body composition from any limitedwhole-body scan is used to estimate approximate limits of trunk. Exact levels are determined by recognition of specific changes in pattern of air and bone. Limiting search for specific patterns only to probable regions avoids unpredictable errors and reduces processing time. Sections above and below trunk are represented by relative distances from these levels. (A) Top of thorax is defined as level where air in expected region of both lungs (black circle) disappears from view on moving from approximate region near top of lung cranially. (B) Bottom of pelvis is defined as level where bone in expected regions of both ischial tuberosities (black circle) disappears from view on moving from pelvic girdle downward.

increase with increasing size of bony pelvis and then drop where the pelvis ended to remain at a relatively constant small area due to the femurs. The exact level where bony pelvis ended was where the sacral tuberosities disappeared from the transaxial section; hence, the only remaining bony structures were the 2 femurs, recognizable by their characteristic location on both sides of the trunk and their roundish configuration.

Relative distance from the top section to top of thorax and bottom of pelvis was defined as the product of the number of sections and slice thickness divided by the body height. The whole coverage of any limited-whole-body scan was defined accordingly by relative distance above top of thorax and below bottom of pelvis, which was used for calculating the ratio of limited-wholebody to whole-body LBM.

\section{Reference of Relative Contribution to Whole-Body Fat Mass}

With the fat mass content and the anatomic location of a limitedwhole-body scan determined, the total body fat mass could be estimated if the relative contribution of fat mass from various anatomic positions were known. Because limited-whole-body scans by definition did not include certain portions of the body, the relationship needed to be established using an external reference model, based on the assumption that people share a similar trend of changing fat composition across different anatomic positions.
To establish such a reference, each whole-body CT dataset was divided into 3 sections, namely the trunk (thorax to pelvis), above the thorax, and below the pelvis. This method was used because the trunk is included in virtually all limited-whole-body PET/CT examinations, whereas the coverage above the thorax and below the pelvis can vary, depending on technical factors and workflow considerations. Also, the required landmarks of top of thorax and bottom of pelvis could be efficiently determined. The whole body was divided into 50 parts along its length, of which 16 were assigned to the trunk, 9 above the thorax, and 25 below the pelvis, to normalize differences in distribution due to variation of body height. Each interval spanned the length of about 1/50 of body height. Fat mass from each CT image was calculated using the aforementioned CT method and grouped into the 1 of the 50 intervals to which it belonged. The result was a plot of distribution of fat mass at different body parts for individual imaging datasets. Pooling the data from individual cases produced the required reference of the relative contributions to total-body fat mass along the length of the whole body, as shown in Figure 3.

In summary, LBM based on the CT data of any limited-wholebody scan could be obtained using the following formula:

$$
\mathrm{LBM}=\text { weight }-\mathrm{FM}_{\mathrm{x} 1-\mathrm{x} 2} / \mathrm{AUC}_{\mathrm{x} 1-\mathrm{x} 2},
$$

where weight $(\mathrm{kg})$ was measured from scale; $\mathrm{x} 1$ and $\mathrm{x} 2$ were the automatically defined relative positions below the pelvis and above the thorax; $\mathrm{FM}_{\mathrm{x} 1}-\mathrm{x} 2(\mathrm{~kg})$ was fat mass calculated from the limited-whole-body CT data; and $\mathrm{AUC}_{\mathrm{x} 1}-\mathrm{x} 2$ was the area under the curve of percentage contribution of fat mass, between position $\mathrm{x} 1$ and position $\mathrm{x} 2$.

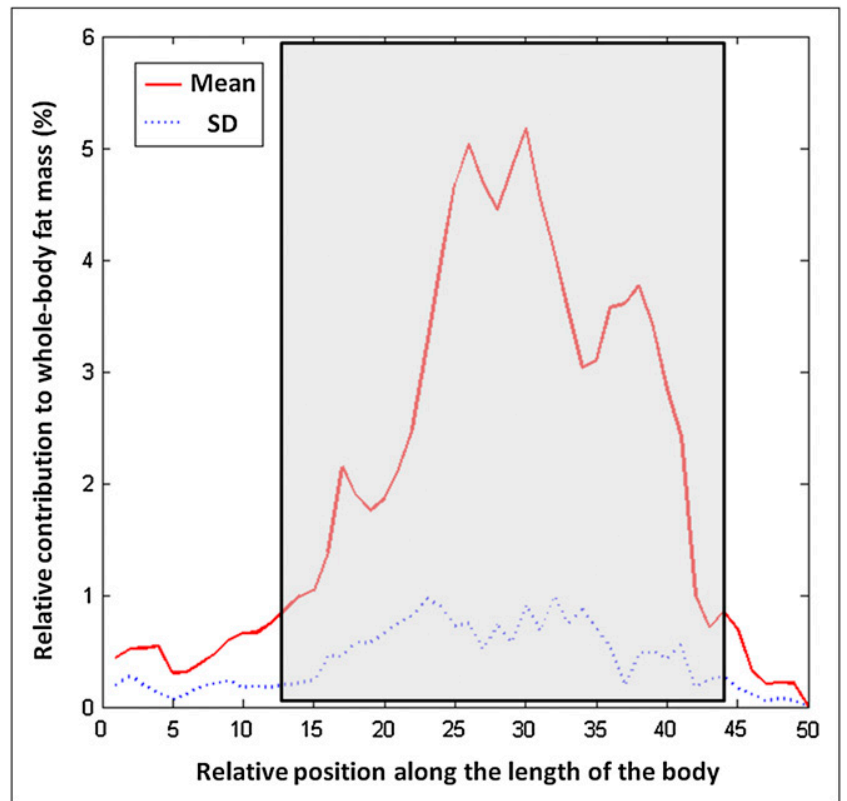

FIGURE 3. Mean and SD of fat mass content as percentage of total body fat mass are plotted against relative locations along length of whole body, from toe at position 1 to head at position 50. This curve was obtained by pooling data from all 18 cases using method stated in text. For any limited-whole-body coverage, for example, as represented by shaded box, area under curve of mean in box equals percentage of total-body fat mass contributed by included anatomic structures. 


\section{Validation of the Computerized Scheme}

For evaluation, the imaging datasets were shortened to different lengths to emulate limited-whole-body scans of different coverages. Each dataset was truncated into 4 different ranges of coverage manually, from the level of the skull vertex or orbital floor superiorly to 5 or $20 \mathrm{~cm}$ below the caudal end of the ischial tuberosities inferiorly. Then, each group of 4 sets of limited-whole-body data from the same individual would be subject to calculation of whole-body LBM using the computerized scheme, designated as LBMb5 (skull base to $5 \mathrm{~cm}$ below pelvis), LBMv5 (skull vertex to $5 \mathrm{~cm}$ below pelvis), LBMb20 (skull base to $20 \mathrm{~cm}$ below pelvis), and LBMv20 (skull vertex to 20 $\mathrm{cm}$ below pelvis). LBM for each case was obtained using the reference of fat distribution established on the basis of data from the rest of the imaging dataset. For example, when the 4 sets of emulated limitedwhole-body data obtained from subject 1 were tested, the reference was established using data from subjects $2-18$. The whole process was repeated for all the datasets, using the jackknife method.

All image processing and analysis tasks, as well as the computerized scheme, were written with MATLAB (The MathWorks, Inc.).

\section{LBM by Predictive Equations}

LBM by the CT method was compared against those derived by 5 different predictive equations; the first 4 have been used in PET studies (Eq. 1 (8), Eq. 2 (1), Eq. 3 (9), and Eq. 4 (10)), whereas the last (Eq. 5 (11)) was included because it takes into account ethnic differences, which might be important because most patients included in this study were ethnic Chinese. LBM calculated on the basis of Eq. 1 was denoted as LBMp1, that of Eq. 2 as LBMp2, and so on.

LBMp1 $=0.32810 \times \mathrm{W}+0.33929 \times \mathrm{H}-29.5336($ for male $)$

$0.29569 \times \mathrm{W}+0.41813 \times \mathrm{H}-43.2933$ (for female)

(Eq. 1)

LBMp2 $=48+1.06 \times(\mathrm{H}-152)$, or $\mathrm{W}$, whichever is less (for male) $45.5+0.91 \times(\mathrm{H}-152)$, or $\mathrm{W}$, whichever is less (for female)

$\mathrm{LBMp} 3=1.1 \times \mathrm{W}-120 \times(\mathrm{W} / \mathrm{H})^{2}($ for male $)$ $1.07 \times \mathrm{W}-148 \times(\mathrm{W} / \mathrm{H})^{2}($ for female $)$

LBMp4 $=\mathrm{W}-\{\mathrm{W} \times[1.2 \times \mathrm{BMI}+(0.23 \times$ age $)-10.8 \times \mathrm{sex}-5.4] / 100\} \quad$ (Eq. 4)

LBMp5 $=\mathrm{W}-[\mathrm{W} \times(76.0-1097.8 / \mathrm{BMI}-20.6 \times \operatorname{sex} / 0.53$

$\times$ age $+95.0 \times$ ethnicity $/$ BMI $-0.44 \times$ ethnicity

$\times$ age $+154 \times$ sex $/$ BMI $+0.034 \times$ sex $\times$ age $)]$,

(Eq. 5)

where $\mathrm{W}$ is weight in $\mathrm{kg}, \mathrm{H}$ is height in $\mathrm{cm}$, body mass index is in $\mathrm{kg} \cdot \mathrm{m}^{-2}$; sex is 0 for female and 1 for male, and ethnicity is 1 for Asian and 0 for other ethnic groups.

LBM derived from whole-body CT data $\left(\mathrm{LBM}_{\mathrm{CT}}\right)$ was used as the gold standard for comparison. LBM obtained using the proposed algorithm and predictive equations was tested for correlations with and differences from the gold standard, using a paired-sample $t$ test. Statistical analyses were performed using the software package PASW Statistics 18 (SPSS Inc.).

\section{RESULTS}

Altogether, there were 26 whole-body PET/CT examinations from 18 patients that fit the selection criteria. The first examinations from each individual formed the image dataset and were made anonymous before being transferred out for subsequent processing and analysis. The examinations were of 10 men and 8 women ranging from 30 to $85 \mathrm{y}$ old, and body mass index was between 16.3 and 29.1. Two patients were Caucasian men, and the rest were Chinese. These examinations covered the range from head to toe instead of the usual skull base to thigh because the wider range was considered necessary for specific clinical considerations. Indications for undergoing PET/CT included melanoma (10), other skin cancers (2), soft-tissue sarcoma (3), lymphoma (1), viral arthritis (1), and hypophosphatemia (1). Fourteen of the examinations incorporated contrast-enhanced CT, whereas the other 4 used unenhanced low-dose CT. The patient characteristics and LBM derived from various methods are listed in Table 1.

The LBM derived from the 5 predictive equations (LBMp1 to LBMp5) showed variable correlations with LBM derived from whole-body CT data $\left(\mathrm{LBM}_{\mathrm{CT}}\right)$, with correlation coefficients $(r)$ ranging from $0.635\left(\mathrm{LBM}_{\mathrm{CT}}\right.$ and LBMp1) to $0.970\left(\mathrm{LBM}_{\mathrm{CT}}\right.$ and LBMp4). LBM produced by the proposed software showed a consistently high correlation with the gold standard, with $r$ ranging from 0.991 $\left(\mathrm{LBM}_{\mathrm{CT}}\right.$ and LBMb5) to 0.998 (LBM $_{\mathrm{CT}}$ and LBMv20).

When tested for paired differences, the predictive equations produced mean differences ranging from $-12.72 \mathrm{~kg}$ (LBMp2) to $1.49 \mathrm{~kg}$ (LBMp1) and SEM ranging from 0.64 (LBMp4) to 2.40 (LBMp1). The software-derived results were again superior, ranging from $0.03 \mathrm{~kg}\left(\mathrm{LBM}_{\mathrm{CT}}\right.$ - LBMv20) to $0.17 \mathrm{~kg}\left(\mathrm{LBM}_{\mathrm{CT}}-\mathrm{LBMb} 5\right)$ in mean differences and $0.03\left(\mathrm{LBM}_{\mathrm{CT}}-\mathrm{LBMv} 20\right)$ to $0.17\left(\mathrm{LBM}_{\mathrm{CT}}-\right.$ LBMb5) in SEM.

The correlations and paired differences are detailed in Table 2.

Compared against the levels of top of thorax and bottom of pelvis labeled by 2 radiologists, the system produced an exact match in 11 of 18 cases; the other 7 cases showed an offset of 2 sections $(0.5 \mathrm{~mm})$ or less in either of the levels. The whole processing time for calculating LBM from each limited-whole-body examination varied between 64 and 104 s, roughly proportional to the distance of coverage, or around $0.23 \mathrm{~s}$ per image section.

\section{DISCUSSION}

Use of the CT method was first introduced in the 1980s by Sjöström et al., who showed that body fat quantified by CT highly correlated with that derived from total-body potassium and was even more reproducible, with less error (12-13). A later study showed excellent agreement between imaging-derived volumes of fat-free skeletal muscles, interstitial fat, and subcutaneous fat and those measured from cadavers (14). CT and MRI have become the most accurate methods for in vivo quantification of total and regional fat tissues (15).

SUV normalized by LBM is commonly used because it has been shown to vary less for people of different body 


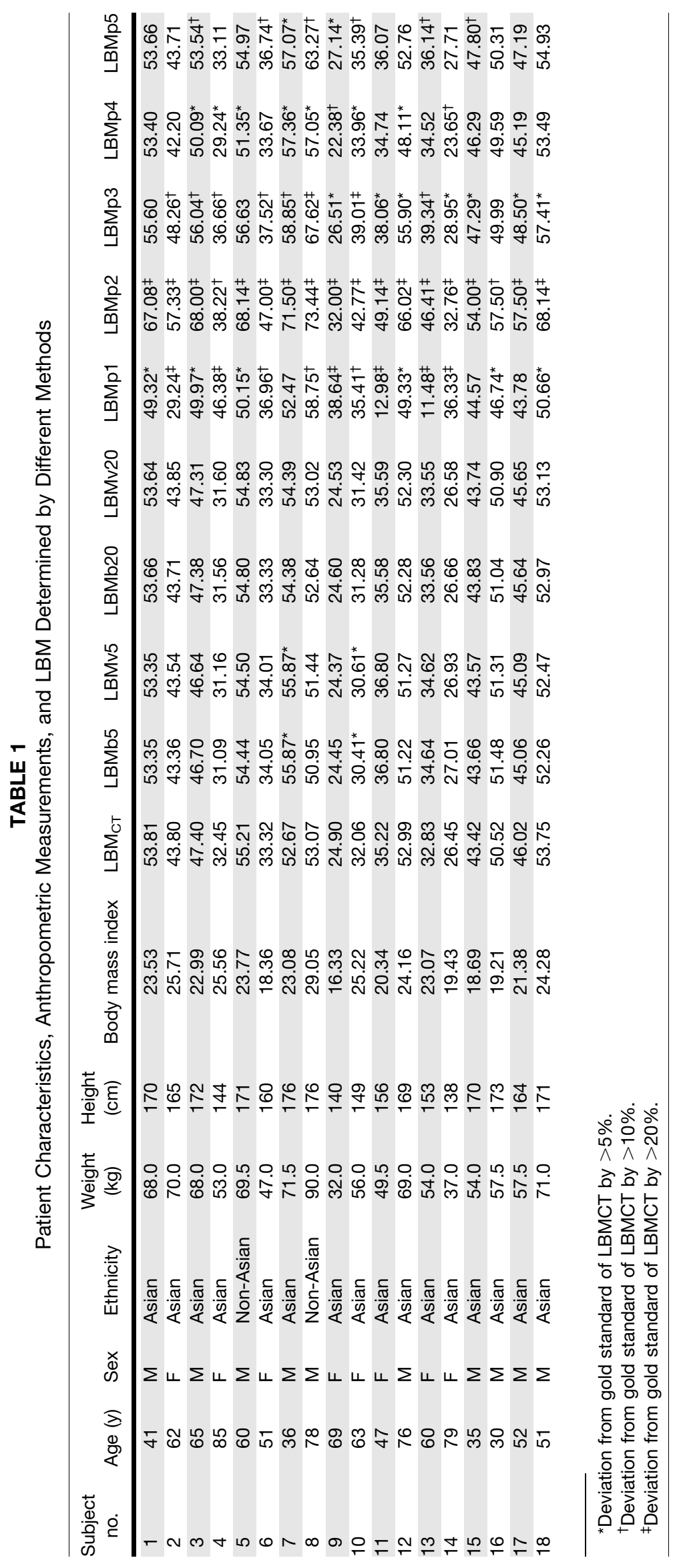

134 The Journal of Nuclear Medicine • Vol. 53 • No. 1 • January 2012 
TABLE 2

Paired Correlation and Paired-Samples Test Between LBM Obtained from Whole-Body CT Data (LBM $\mathrm{CT})$ and LBM Obtained from Proposed Method (LBMb5, LBMv5, LBMb20, LBMv20) and Predictive Equations (LBMp1 to LBMp5)

\begin{tabular}{|c|c|c|c|c|c|c|}
\hline \multicolumn{3}{|c|}{ Paired-samples correlations } & \multicolumn{4}{|c|}{ Paired-samples test } \\
\hline Pair & $\begin{array}{l}\text { Correlation } \\
\text { coefficient }\end{array}$ & Significance & Pair & Mean $(\mathrm{kg})$ & SEM (kg) & $\begin{array}{l}\text { Significance } \\
\text { (2-tailed) }\end{array}$ \\
\hline LBM $_{\mathrm{CT}}$ and LBMb5 & 0.991 & $<0.001$ & LBM $_{\mathrm{CT}}-\mathrm{LBMb5}$ & 0.17 & 0.33 & 0.615 \\
\hline LBM $_{\mathrm{CT}}$ and LBMv5 & 0.992 & $<0.001$ & LBM $_{\mathrm{CT}}-\mathrm{LBMv5}$ & 0.13 & 0.31 & 0.686 \\
\hline $\mathrm{LBM}_{\mathrm{CT}}$ and LBMb20 & 0.998 & $<0.001$ & $\mathrm{LBM}_{\mathrm{CT}}-\mathrm{LBMb} 20$ & 0.06 & 0.15 & 0.723 \\
\hline $\mathrm{LBM}_{\mathrm{CT}}$ and $\mathrm{LBMv20}$ & 0.998 & $<0.001$ & LBM $_{C T}-$ LBMv20 & 0.03 & 0.14 & 0.831 \\
\hline LBM $_{\mathrm{CT}}$ and LBMp1 & 0.635 & .005 & $\mathrm{LBM}_{\mathrm{CT}}-\mathrm{LBMp} 1$ & 1.49 & 2.40 & 0.544 \\
\hline LBM $_{\mathrm{CT}}$ and LBMp2 & 0.959 & $<0.001$ & LBM $_{C T}-$ LBMp2 & -12.72 & 1.08 & $<0.001$ \\
\hline LBM $_{\mathrm{CT}}$ and LBMp3 & 0.954 & $<0.001$ & LBM $_{C T}-$ LBMp3 & -4.35 & 0.80 & $<0.001$ \\
\hline LBM $_{\text {CT }}$ and LBMp4 & 0.970 & $<0.001$ & $\mathrm{LBM}_{\mathrm{CT}}-\mathrm{LBMp} 4$ & 0.20 & 0.64 & 0.759 \\
\hline LBM $_{\mathrm{CT}}$ and LBMp5 & 0.967 & $<0.001$ & LBM $_{\mathrm{CT}}-\mathrm{LBMp} 5$ & -2.31 & 0.650 & .002 \\
\hline
\end{tabular}

weights, is comparable between individuals of different body habitus, and is of a range similar to SUV normalized by body weight $(1,9)$.

Strictly speaking, LBM is slightly different from fat-free mass, in that the former but not the latter includes fat in cell membranes (16). Although most predictive equations were derived for determination of fat mass (or the readily derived fat-free mass as body weight-fat mass), virtually all previous studies relating to methods of determining LBM for SUV normalization used the terms interchangeably (4). Because the CT method can resolve only macroscopically visible volumes of fat, not fat in cell membranes from fat within soft tissues, the CT method calculates LBM rather than fat-free mass.

Apart from Equation 1, the other predictive equations produced high correlations with the gold standard used in this study. Equation 2, although showing a high correlation coefficient, consistently overestimated LMB in all cases by $13.8 \%-43.5 \%$. This overestimation was recognized by the same group of investigators who proposed Equation 2, who later suggested use of Equation 3 instead. However, Equation 3 still showed considerable variability; almost half the cases showed an error of more than $10 \%$, with 2 showing an error of more than $20 \%$. Even for the best of the predictive equations used in this study, Equation 4, half the cases still showed an error of more than 5\%, with 2 showing an error of more than $10 \%$. Equation 5, incorporating adjustments for racial differences that should apparently be more applicable for the current sample that included far more Asians, did not do better than Equation 4 in this study: 8 cases showed an error of more than $5 \%$, with 6 showing an error of more than $10 \%$. The results implied that the LBM-normalized SUV obtained from any of these equations would be similarly variable.

The fact that different predictive equations can produce quite variable LBMs for the same individuals, as illustrated by the results of this study, should prompt the imaging community to arrive at a consensus as to which particular equation is preferred. Because display workstations from different vendors do not necessarily apply the same formula in their LBM calculations, the same imaging dataset may produce a different readout of LBM-normalized SUV. At the very least, use of any particular equation in the display workstation should be explicitly described, so that cross-platform comparison is possible with appropriate adjustments.

All the predictive equations for LBM were regression formulae derived from different reference methods on their study population (17-19). It is questionable if these equations can be applied to individuals who may have a body composition or other related characteristics different from the original study population from which the formulae were derived. For example, predictive equations derived from a population of normal body habitus may not apply to obese patients (20) and those derived from a specific ethnic group may not apply to a different ethnic group (11). One further problem is that chronic disease can be associated with a predominant loss of LBM rather than body weight, due to wasting of skeletal muscles (16), rendering predictive equations derived from a population of normal health inaccurate.

One major theoretic advantage of the proposed method over predictive equations is that it is based primarily on the directly measured LBM, albeit incomplete, of an individual, rather than on an assumed similarity between the subject and some specific study population. The results indicated that the proposed method was more accurate and consistent than any of the tested predictive equations, reaching a correlation coefficient of more than 0.99 even in the shortest coverage tested, which was considered unusually short. More importantly, only in the 2 shortest ranges were there 2 cases that showed an error of slightly more than $5 \%$.

This computerized scheme obviously works for only PET/CT, not PET-alone, imaging data. But this limitation is fading because PET/CT has virtually supplanted PET-only scanners because of its superior sensitivity and specificity 
and enhanced throughput. Therefore, CT data are almost always readily available for accurate determination of LBM, which can subsequently be used for more consistent SUV normalization.

The imaging data in the current project included both normal-dose contrast-enhanced CT and low-dose unenhanced CT. Although the low-dose CT images were noisier, the noise did not seem to affect the accuracy of segmentation results on visual inspection, at least partly because of the denoising procedure that was applied to all CT datasets in the program and that removed small, focal variations in signal intensity. Because of the small sample size, potential differences between results obtained from normal-dose versus low-dose CT could not be defined.

It is obvious that fat mass distribution varies between people of different body habitus. For example, people having truncal obesity would have a larger proportion of fat mass coming from the trunk, and a commensurately lower proportion from the limbs, than a normal-weight population. Limited by the small sample size and patient heterogeneity (e.g., age, sex, and body mass index) in the current imaging dataset, the reference so produced, as illustrated in Figure 3, may not be the most robust and may not be equally applicable to different populations. Still, the results showed the variation to be largest (largest SD) in the center of the trunk, decreasing significantly toward both ends. Therefore, the algorithm still produced accurate results in the usual, albeit variable, coverage used in limited-whole-body PET/CT examinations. Increasing coverage toward both ends should produce a more accurate relative contribution of fat mass and hence a more accurate estimation of whole-body LBM ultimately, as was also supported by the finding that longer coverage of the test data produced more accurate estimations of true wholebody LBM. It is anticipated that the robustness and accuracy of the proposed method can be further improved by amassing imaging data from a large number of subjects to produce a stable reference model of fat mass distribution at different anatomic levels. Acquiring such a database may require contributions from multiple centers or a center at which whole-body coverage is routinely performed.

The proposed method was more accurate than commonly used predictive equations. Intuitively, more accurate measurement of LBM should produce more accurate SUVs normalized by LBM. But the clinical benefit cannot be realized if the LBM-normalized SUV so derived is not more reproducible. A previous study that measured the SUV of 70 cancer-free patients over 2 occasions suggested that neither SUV normalized by LBM nor SUV normalized by body surface area improved intraindividual variability at different time points (21). That study used E3 for calculating LBM. Whether use of the proposed method would result in better reproducibility for individual subjects using LBM-normalized SUV over other normalization methods, especially for cancer patients, who might show a drop in LBM over time, remains to be further investigated.

\section{CONCLUSION}

An automatic computerized method for evaluation of LBM based on CT data from limited-whole-body coverage was developed. The calculated LBM was more accurate than results obtained by predictive equations that have been routinely used in clinical PET/CT examinations. It is anticipated that this method can improve the accuracy and consistency of LBM-adjusted SUV.

\section{DISCLOSURE STATEMENT}

The costs of publication of this article were defrayed in part by the payment of page charges. Therefore, and solely to indicate this fact, this article is hereby marked "advertisement" in accordance with 18 USC section 1734.

\section{ACKNOWLEDGMENT}

This work was funded by the University of Hong Kong. No other potential conflict of interest relevant to this article was reported.

\section{REFERENCES}

1. Zasadny KR, Wahl RL. Standardized uptake values of normal tissues at PET with 2-[fluorine-18]-fluoro-2-deoxy-D-glucose: variations with body weight and a method for correction. Radiology. 1993;189:847-850.

2. Adams MC, Turkington TG, Wilson JM, Wong TZ. A systematic review of the factors affecting accuracy of SUV measurements. AJR. 2010;195: $310-320$.

3. Wahl RL, Jacene H, Kasamon Y, Lodge MA. From RECIST to PERCIST: evolving considerations for PET response criteria in solid tumors. J Nucl Med. 2009;50(suppl 1):122S-150S

4. Erselcan T, Turgut B, Dogan D, Ozdemir S. Lean body mass-based standardized uptake value, derived from a predictive equation, might be misleading in PET studies. Eur J Nucl Med Mol Imaging. 2002;29:1630-1638.

5. Kullberg J, Brandberg J, Angelhed JE, et al. Whole-body adipose tissue analysis: comparison of MRI, CT and dual energy x-ray absorptiometry. Br J Radiol. 2009; 82:123-130.

6. Chowdhury B, Sjostrom L, Alpsten M, Kostanty J, Kvist H, Lofgren R. A multicompartment body composition technique based on computerized tomography. Int J Obes Relat Metab Disord. 1994;18:219-234.

7. Müller MJ, Bosy-Westphal A, Kutzner D, Heller M. Metabolically active components of fat-free mass and resting energy expenditure in humans: recent lessons from imaging technologies. Obes Rev. 2002;3:113-122.

8. Velasquez LM, Boellaard R, Kollia G, et al. Repeatability of ${ }^{18}$ F-FDG PET in a multicenter phase I study of patients with advanced gastrointestinal malignancies. J Nucl Med. 2009;50:1646-1654.

9. Sugawara Y, Zasadny KR, Neuhoff AW, Wahl RL. Reevaluation of the standardized uptake value for FDG: variations with body weight and methods for correction. Radiology. 1999;213:521-525.

10. Pieterman R, Willemsen A, Appel M, et al. Visualisation and assessment of the protein synthesis rate of lung cancer using carbon-11 tyrosine and positron emission tomography. Eur J Nucl Med Mol Imaging. 2002;29: $243-247$.

11. Gallagher D, Heymsfield SB, Heo M, Jebb SA, Murgatroyd PR, Sakamoto Y. Healthy percentage body fat ranges: an approach for developing guidelines based on body mass index. Am J Clin Nutr. 2000;72:694-701.

12. Sjöström L, Kvist H, Cederblad A, Tylen U. Determination of total adipose tissue and body fat in women by computed tomography, 40K, and tritium. Am J Physiol. 1986;250:E736-E745.

13. Sjöström L. A computer-tomography based multicompartment body composition technique and anthropometric predictions of lean body mass, total and subcutaneous adipose tissue. Int J Obes. 1991;15(suppl 2):19-30.

14. Mitsiopoulos N, Baumgartner RN, Heymsfield SB, Lyons W, Gallagher D, Ross R. Cadaver validation of skeletal muscle measurement by magnetic resonance imaging and computerized tomography. J Appl Physiol. 1998;85: $115-122$. 
15. Mattsson S, Thomas BJ. Development of methods for body composition studies. Phys Med Biol. 2006;51:R203-R228.

16. Roubenoff R, Kehayias JJ. The meaning and measurement of lean body mass. Nutr Rev. 1991;49:163-175.

17. Hume R. Prediction of lean body mass from height and weight. J Clin Pathol. 1966;19:389-391.

18. James WPT. Research on Obesity: A Report of the DHSS/MRC Group. London, U.K.: HMSO; 1976.
19. Deurenberg P, Weststrate JA, Seidell JC. Body mass index as a measure of body fatness: age- and sex-specific prediction formulas. Br J Nutr. 1991;65:105-114.

20. Fuller NJ, Sawyer MB, Elia M. Comparative evaluation of body composition methods and predictions, and calculation of density and hydration fraction of fat-free mass, in obese women. Int J Obes Relat Metab Disord. 1994;18: 503-512.

21. Paquet N, Albert A, Foidart J, Hustinx R. Within-patient variability of ${ }^{18}$ F-FDG: standardized uptake values in normal tissues. J Nucl Med. 2004;45:784-788. 